\title{
B cell lymphoma of the thymus and salivary gland
}

\author{
C Di Loreto, L Mariuzzi, A De Grassi, C A Beltrami
}

\begin{abstract}
A case of primary low grade B cell lymphoma of the salivary gland associated with a low grade B cell lymphoma of the thymus and involvement of the skin is reported. The lesions in the salivary gland and in the thymus showed the typical features of a lymphoma arising from the mucosa associated lymphoid tissue (MALT) and comprised lymphatic follicles, centrocyte-like (CCL) cells and lymphoepithelial lesions. Immunohistochemistry and Southern blot analysis supported the hypothesis that these lesions can originate from the same cellular clone. These findings confirm the occurrence of low grade B cell MALT lymphoma in the thymus and the possibility of spread of MALT lymphoma to other mucosal sites.

(F Clin Pathol 1996;49:595-597)
\end{abstract}

Keywords: low grade B cell lymphoma, MALT, salivary gland, thymus.

The malignant lymphomas of mucosa associated lymphoid tissue (MALT) are variants of $B$ cell lymphomas and occur in organs embryologically derived from the foregut. ${ }^{1-3}$ Low grade lymphoma mimics the organisation of MALT, is normally present in the gastrointestinal tract and the neonatal lung, or is acquired in inflammatory diseases of the salivary and thyroid glands. ${ }^{1}$ Low grade, B cell MALT lymphomas are usually indolent and are localised at presentation; nevertheless, they may occur in other mucosal sites. ${ }^{4}$

Here, we report a case of low grade, B cell lymphoma with the morphological features of low grade MALT lymphoma and localised to the submandibular salivary gland, thymus and skin. \section{Medical and \\ Morphological \\ Research, \\ University of Udine, \\ Italy \\ C Di Loreto \\ L Mariuzzi \\ C A Beltrami}

Department of

Department of Clinical and Experimental Medicine and Pathology, University of Udine A De Grassi

Correspondence to: Dr C Di Loreto,

Ospedale S. Maria della

Misericordia

33100 Udine, Italy. \begin{abstract}
right and left mediastinal pleura, was removed. Macroscopically, it was $10 \mathrm{~cm}$ at its maximum diameter and weighted $100 \mathrm{~g}$. The cut surface was spongy with numerous fluid filled cysts up to $3 \mathrm{~cm}$ in diameter.

A 51 year old man presented with swelling of anterior mediastinal mass that, on comput tomography scanning, was found to arise in the
\end{abstract}

The patient did not receive further treatment. One year later, he developed multiple livid skin plaques on his head, one of which was biopsied.

\section{Methods}

Fresh tissue samples from the submandibular gland and from the mediastinal tumour were snap frozen. Multiple blocks from the salivary gland, thymus and skin specimens were fixed in $10 \%$ buffered formalin and stained with haematoxylin and eosin. The Congo red method was used to detect amyloid in the skin sections.

Paraffin wax sections were stained immunohistochemically by the avidin-biotin peroxidase complex (ABC) method ${ }^{5}$ using the following antibodies: anti- $\kappa$, anti- $\lambda$, anti-IgA, anti-IgM, anti-IgG, anti-IgD for immunoglobulins, CD20 (L26) for B cells, CD21 (1F8) for dendritic follicular cells and B cells, and CD3 and CD45RO (UCHL1) for T cells. All antibodies were purchased from Dako, Glostrup, Denmark.

Southern blotting was carried out on genomic DNA extracted from frozen salivary gland and thymus tissue to detect immunoglobulin chain rearrangements, if any, as described previously. ${ }^{6}$ Genomic DNA was digested with $10 \mathrm{U} / \mathrm{ml}$ EcoRI, BamHI and HindIII for 12 hours at $37^{\circ} \mathrm{C}$, electrophoresed in a $0.8 \%$ agarose gel and transferred to nylon filters (Qiagen, Chatsworth, California, USA) by Southern blotting. The IGKC probe, spanning a constant region of 2.5 kilobases of the immunoglobulin $\kappa$ chain gene ${ }^{7}$ and the IGLC2 probe, spanning a constant region of 3.5 kilobases of the $\lambda$ chain gene, ${ }^{8}$ were labelled with $\left[{ }^{32} \mathrm{P}\right]-\mathrm{dCTP}$ using a commercially available nick translation kit (Boehringer Mannheim, Mannheim, Germany). The membranes were then subjected to high stringency washes and autoradiographed on Kodak XAR-5 film for five days at $-80^{\circ} \mathrm{C}$.

\section{Results}

On microscopy the salivary gland was characterised by a lymphoid infiltrate containing several epimyoepithelial islands, which obliterated the overall structure of the gland. The lymphoid infiltrate was composed of a broad proliferation of small cells with heterochromatic, irregularly shaped nuclei resembling centrocytes (centrocyte-like (CCL) cells). These typical CCL cells were located in the vicinity of and had infiltrated and partially disrupted the epimyoepithelial islands. Blast-like cells with nucleoli were present; plasma cells were rarely seen. Numerous follicles showing variable degrees of distortion were also observed. 


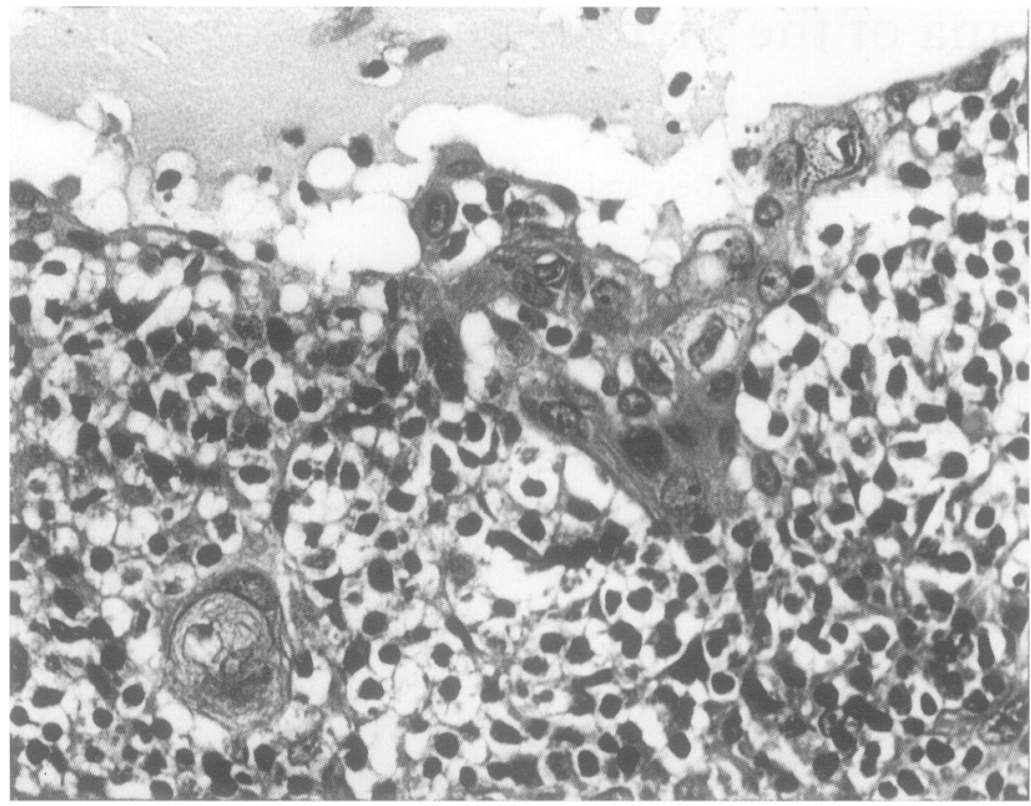

Figure 1 Thymus. Diffuse lymphoid infiltrate showing cells with clear cytoplasm and well defined margins. These cells have invaded the epithelium lining a cyst, forming a lymphoepithelial lesion. Scattered blasts and plasma cells are also present. A residual Hassall's corpuscle is evident. (Haematoxylin and eosin, $\times 250$.)

The architecture of the thymus was obscured by the presence of a dense lymphoid infiltrate and cysts. The lymphoid infiltrate was predominantly composed of sheets of intermediately sized cells with irregular nuclei, sharp cell borders and clear cytoplasm, the so-called clear cell variant of the CCL cell. Some larger, nucleolated lymphoid cells were scattered throughout the infiltrate. Numerous clusters of plasma cells merged and showed a morphological continuum with underlying CCL cells. Foci of lymphatic follicles with germinal centres were also seen. The cysts comprised a large part of the lesion, were lined by squamous epithelium and contained clear

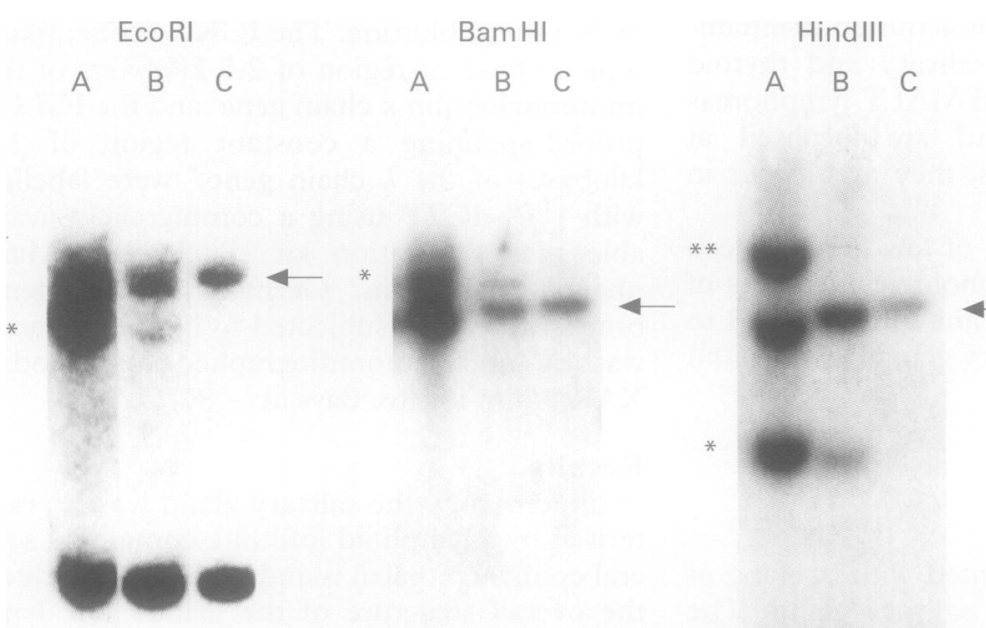

Figure 2 Southern blot analysis showing immunoglobulin gene rearrangement in DNA extracted from the $(A)$ salivary gland, $(B)$ thymus and $(C)$ and bone marrow stromal cells. Clonally rearranged bands are present in DNA from the thymus and salivary gland. The arrows show germ line bands and asterisks show the rearranged bands. Digestion of DNA with HindIII showed one clonally rearranged band $\left(^{*}\right)$ in tissue from the salivary gland and thymus and a second band $\left(^{*}\right)$ in tissue from the salivary gland only. This observation suggests that the neoplastic cells in the thymus may represent a subclone of the neoplastic cells from salivary gland, characterised by the lack of one restriction site for HindIII. Contaminating normal cells are arrowed. eosinophilic material. The epithelium of these cysts was heavily infiltrated by CCL cells, forming "lymphoepithelial lesions". Residual Hassall's corpuscles invaded by clusters of lymphoid cells were also present (fig 1 ).

The skin biopsy had a dermal infiltrate with a prevalent perivascular distribution. This infiltrate contained lymphoid cells with irregular nuclei, clear cytoplasm and many plasma cells. Deposits of eosinophilic hyaline material showing, on staining with Congo red, green birefringence in polarised light were observed in the derma.

IMMUNOHISTOCHEMICAL AND GENETIC FINDINGS The lymphoid population in the salivary gland consisted of B cells (CD20 positive). Blast cells were positive for the $\kappa$ light chain and the $\alpha$ heavy chain.

In the thymus, the lymphoid infiltrate and CCL cells in the lymphoepithelial lesions were CD20 positive, which is suggestive of a $B$ cell phenotype. The neoplastic population expressed the $\kappa$ light chain. (Staining was mainly restricted to the perinuclear space of CCL cells, but in the larger cells the whole cytoplasm stained.) Cells expressing light chains also expressed the $\alpha$ heavy chain; these cells merged with the monotypic plasma cell population expressing $\operatorname{Ig} \mathrm{A} / \kappa$.

In the skin biopsy specimen lymphoid cells were CD20 positive and plasma cells showed an $\operatorname{IgA} / \kappa$ monoclonality.

In the Southern blots of DNA extracted from salivary gland and thymus (fig 2, lanes A and $B$, respectively), identical, clonally rearranged bands representing the immunoglobulin $K$ constant region were present.

Digestion with HindIII showed a second band present in salivary gland samples only, suggesting that the neoplastic cells in the thymus may represent a subclone lacking of one of the restriction sites for HindIII (fig 2).

\section{Discussion}

Three cases of low grade, B cell MALT lymphoma arising in the thymus have, to our knowledge, been described previously ${ }^{39}$ and one case has been reported in association with a benign lymphoepithelial lesion of the minor salivary glands in a patient with Sjögren's syndrome. ${ }^{9}$ In our case B cell lymphoma of the thymus was associated with B cell lymphoma of the salivary gland, both showing morphology consistent with low grade, B cell MALT lymphoma. Skin lesions appeared subsequently.

In the present case the CCL cells expressed $\kappa$ light chains on immunohistochemical analysis. This was confirmed by genotypic analysis, which revealed rearrangement of the immunoglobulin $\kappa$ chain gene. The presence of $B$ lymphoid cells in the thymus is well documented; lymphatic follicles are present in the thymus in patients with myasthenia gravis and it has been suggested that a benign condition of the thymus, similar to myoepithelial sialadenitis (MESA), is occasionally a precursor of MALT lymphoma. Finally, Hassall's corpuscle epithelium, because of its ability to synthesise 
hormonal peptides, can be considered mucosal in nature. These observations may explain the primitive origin of the MALT lymphoma in the thymus. The morphological features of the submandibular gland-that is, expansion of the lymphoid infiltrate and the presence of blast forms of CCL cells, are diagnostic of a lymphomatous process and genetic analysis demonstrated a $\kappa$ chain rearrangement. The morphological and genotypic analyses are consistent with a diagnosis of B cell low grade malignant lymphoma of the salivary gland ${ }^{2}$ exhibiting features of a MALT lymphoma. As the salivary gland normally lacks lymphoid tissue, the MALT lymphoma may have originated from tissue involved by MESA. However, there was no evidence of underlying MESA in our patient. The infiltrate of the skin was composed of a few clear CCL cells and many monotypic IgA/ $\kappa$ plasma cells. Plasma cell differentiation was associated with amyloid deposits. The presence of CCL cells and the monoclonality of the plasma cells suggest that the cutaneous infiltrate originated via the same lymphomatous process.

It is of note that the lesions in the salivary gland and the thymus were histologically similar and also had identical clonally rearranged bands. The morphological features of MALT lymphoma in the salivary gland and the thymus have been reported previously. ${ }^{3}$ It is possible that multiple mucosal sites can be involved by MALT lymphoma or that the lymphoma can spread from the salivary gland to the thymus. This pattern of spread probably depends on the specific circulation pathways and homing characteristics of the lymphocytes present. We can put forward two hypotheses: (1) that the B lymphocytes of the thymus and salivary gland are closely related; and (2) that B lymphocytes can move between these two organs. Similarly, the lymphomatous localisation in the skin could have resulted from peripheral spread of the tumours and could be related, at the least in part, to lymphocyte-endothelial cell recognition. Skin directly exposed to the external environment may be a site for so-called acquired B cell, skin associated lymphoid tissue. Cutaneous B cell lymphomas have numerous aspects in common with MALT lymphoma, not least being originated from lymphoid tissue. ${ }^{10}$

In conclusion, our case is unique because the patient has evidence of MALT lymphoma in the salivary gland and in the thymus showing the same genotypic pattern, with further localisation in the skin. This confirms the possibility of multiple mucosal sites involved by lymphoma or the spread of a MALT lymphoma from one mucosal site to another, including the skin.

1 Isaacson PG, Spencer J. Malignant lymphoma of mucosaassociated lymphoid tissue. Histopathology 1987;11:44562 .

2 Hyjek E, Smith WJ, Isaacson PG. Primary B cell lymphoma of salivary gland and its relationship to myoepithelial sialadenitis (MESA). Hum Pathol 1988;19:766-76.

3 Isaacson PG, Chan JKC, Tang C, Addis BJ. Low-grade B-cell lymphoma of mucosa-associated lymphoid tissue arising in the thymus. A thymic lymphoma mimicking myoepithelial sialadenitis. Am ₹ Surg Pathol 1990;14:34251 .

4 Li G, Hansmann ML, Zwingers T, Lennert K. Primary ymphomas of the lung: morphological, immunohistochemical and clinical features. Histopathology 1990 16:519-33.

5 Norton AJ, Isaacson PG. Detailed phenotypic analysis of B-cell lymphoma using a panel of antibodies reactive in B-cell lymphoma using a panel of antibodies reactive in 1987; 128:225-40.

6 Sambrook J, Fritsch EF, Maniatis T. Molecular cloning. $A$ laboratory manual. 2th edn. Cold Spring Harbor: Cold Spring Harbor Laboratory Press, 1989.

7 Hieter PA, Max EE, Seidman JG, Marzel JV Jr, Leder P. Cloned human and mouse kappa immunoglobulin conCon segments. Cell 1980;22:197-207.

8 Hieter PA, Hollis GF, Horsmeyer SJ, Waldmann TA, Leder P. Clustered arrangement of immunoglobulin lambda constant region genes in man. Nature 1981;294:536-40.

9 Takagi N, Nakamura S, Yamamoto K, Kunishima K, Takagi I, Suyama $M$, et al. Malignant lymphoma of mucosaassociated lymphoid tissue arising in the thymus of a patient with Sjogren's syndrome. Cancer 1992;69:134755.

10 Gianotti B, Santucci M. Skin-associated lymphoid tissue (SALT)-related B-cell lymphoma (primary cutaneous B-cell lymphoma). Arch Dermatol 1993;129:353-5.

\section{Kappa statistics as indicators of quality assurance in histopathology and cytopathology}

\section{Department of Pathology, University of Sheffield Medical School, Beech Hill Road, Sheffield S10 2UL}

email:

s.s.cross@sheffield.ac.uk

Accepted for publication 21 March 1996

\author{
S S Cross
}

positive rates. It was found that the level at which the upper $95 \%$ confidence interval for the kappa statistic fell below 1 was an insensitive method of detecting unsatisfactory performance as at that level the false positive rate was unacceptably high $(>1 \%)$ for all populations of specimens less than 800 in number. Either large populations of samples are required in quality assurance schemes which use kappa statistics (which may well be impractical) or 